\title{
IDENTIFICACIÓN DE MAMIIFEROS, AVES, PECES Y REPTILES COMO ESTRATEGIA PARA EL FORTALECIMIENTO DE LAS COMPETENCIAS DEL ÁREA DE CIENCIAS NATURALES
}

\author{
Mammals, birds, fish and reptiles identification as a strategy for \\ strengthening of the skills of the natural sciences area
}

\section{Artículo de Investigación}

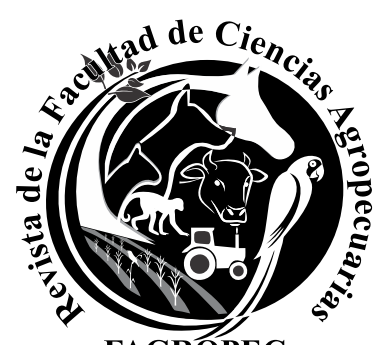

FAGROPEC

Recibido 21 de enero de 2019. Aceptado 15 de marzo de 2019.

${ }^{1}$ Biólogo, Esp, Mgs. Phd. en Ciencias. Docente Secretaría de Educación Departamental del Caquetá.

${ }^{2} \mathrm{MVZ}$, Lic. Inglés, Esp. FYEP. Content Developer. Universidad del Istmo.

\section{Como citar:}

PARRA-HERRERA, J.P. y ESCUDEROSÁNCHEZ, O.A.. Identificación de mamíferos, aves, peces y reptiles como estrategia para el fortalecimiento de las competencias del área de ciencias naturales. En: Revista Facultad Ciencias Agropecuarias - FAGROPEC. Universidad de la Amazonia, Florencia Caquetá. Volumen 11 enero-junio, 2019. Pp. 3342. ISSN-Revista en Línea: 2539-178X

*Autor para correspondencia: juanfauna@gmail.com
Juan Pablo Parra - Herrera ${ }^{1 *}$ y Oscar Andrés Escudero - Sánchez ${ }^{2}$

\section{RESUMEN}

El conocimiento detallado del número de especies presentes en un área geográfica es fundamental para la realización de acciones y programas para el uso, protección y conservación biológica. En este sentido, los inventarios faunísticos han demostrado ser de gran utilidad para el conocimiento y uso de la biodiversidad. Partiendo de lo anterior se realizó un inventario de biodiversidad con estudiantes de la Institución Educativa Rural Platanillo de la sede el Vergel en el municipio de san José del Fragua-Caquetá. Donde se utilizó como método de muestreo metodologías no invasivas como puntos de observación para aves, trampas cámara y huellas, búsqueda intensiva en anfibios y reptiles y redes de arrastre en peces. Esta investigación arrojo como resultado un total de 81 especies registradas. El grupo taxonómico con el mayor número de especies fue el de aves con 54 especies, seguido de los mamíferos y en último lugar anfibio, reptil y peces. El uso de la investigación contribuyo no solo a conocer las especies de la región si no en el cambio de actitud de los estudiantes, favoreciendo significativamente los procesos de enseñanza aprendizaje. Lo anterior, se evidencia en los resultados de investigación obtenidos por los estudiantes donde se evidencio un fortalecimiento en competencias del área de ciencias naturales en los entornos vivo, físico y químico, a través de la investigación, convirtiéndose en una aproximación al listado actualizado de biodiversidad faunística para el sur de la amazonia Caqueteña.

Palabras claves:

Biodiversidad; Estudiantes; Inventario.

\section{ABSTRACT}

Detailed knowledge of the number of species present in a geographic area is essential to carry out actions and programs for the use, protection, and biological conservation. In this sense, fauna inventories have proven to be very useful for the knowledge and use of biodiversity. Based on the above, a biodiversity inventory was carried out with students from Institución Educativa Rural Platanillo sede el Vergel in the municipality of San José del Fragua-Caqueta. Where non-invasive methodologies such as observation points for birds, camera traps and footprints, intensive search in amphibians and reptiles, and trawls in fish were used as a sampling method. This research resulted in a total of 81 registered species. The taxonomic group 
with the highest number of species was birds with 54 species, followed by mammals, and lastly amphibians, reptiles, and fish. The use of research contributed not only to know the species of the region but also in the change of attitude of the students, significantly favoring the teaching-learning processes. The foregoing is evidenced in the research results obtained by the students, where a strengthening of competencies in the area of natural sciences in the living, physical and chemical environments as evidenced through research, becoming an approximation to the updated list of fauna biodiversity for the southern Amazon of Caquetá.

Key words:

Inventory; Biodiversity, Students

\section{INTRODUCCIÓN}

La IER Platanillo se encuentra ubicada en el piedemonte de la Cordillera Oriental y se sitúa al nororiente del Municipio de San José del Fragua departamento del Caquetá en la zona de amortiguación al Parque Nacional Natural Alto Fragua Indi Wasi. Esta zona se caracteriza por una gran diversidad biológica pero enfrenta problemas de colonización, deforestación y el aprovechamiento de la tierra para sistemas convencionales, producción ganadera y cultivos ilícitos, provocando transformación de los ecosistemas naturales, cuenta con grandes vacíos del conocimiento de su biodiversidad y una fuerte falencia en la enseñanza de la investigación en las ciencias naturales que es fundamental en la conservación de los ecosistemas por los pobladores locales, la falta de apropiación de los recursos faunísticos por parte de los estudiantes es una problemática vista día tras día afecta a la sede el Vergel, los alumnos apenas logran reconocer las características de los seres vivos y tienen limitaciones en establecer relaciones con el ambiente; tampoco pueden representar algunos eventos naturales, taxonomía de animales silvestre y realizar investigación.

Aunque los niños que habitan la zona rural observan diariamente los alrededores de su entorno, desconocen las especies animales y vegetales características de su región, la importancia de estas y de los ecosistemas que los rodean (MEN, 2015). Lo anterior se atribuye en parte, a que la formación en ciencias de la naturaleza no ha sido orientada desde las necesidades de las comunidades rurales, pues por lo general los materiales han sido diseñados para los que habitan en lugares donde la diversidad biológica está conformada por otros organismos y está influenciada por factores climáticos completamente diferentes a los de nuestra región amazónica (Sánchez, 2015).

Es en este sentido, que la investigación como herramienta en la formación de competencias científicas, puede ofrecer gran cantidad de posibilidades para mejorar los procesos de enseñanza aprendizaje que se llevan a cabo dentro y fuera del aula de clase, creando entornos de aprendizaje lúdicos e interactivos (Deruyttere, et al. 1997). Por tal motivo, la presente investigación busca fortalecer las competencias del área de ciencias naturales en los entornos vivo, físico y químico, a través de la investigación. El conocimiento de la diversidad biológica favorece los propósitos de la educación ambiental, ya que esta busca producir ciudadanos conscientes de su entorno biofísico y los problemas asociados a este, lo cual se logra sobre la base del conocimiento de los ecosistemas (Hilty, 2010) 
El presente proyecto se enmarco en los procesos generales de las Ciencias Naturales que define el Ministerio de Educación de Colombia (2015) sobre los cuales se evalúa el uso comprensivo del conocimiento científico, la explicación de fenómenos y la indagación. El uso de la investigación como herramienta en la formación de competencias científicas pueden ofrecer gran cantidad de posibilidades para mejorar los procesos de enseñanza aprendizaje que se llevan a cabo dentro y fuera del aula de clase, creando entornos de aprendizaje lúdicos e interactivos. Por tal motivo, la presente investigación busco fortalecer las competencias del área de ciencias naturales en los entornos vivo, físico y químico, a través de la investigación (Geilfus, 1996, Graipel, 2003).

\section{MATERIALES Y MÉTODOS}

Área de estudio: La sede el Vergel se encuentra ubicada a $1^{\circ} 21.3420 \mathrm{~N}$ Latitud y $75^{\circ} 57.646$ con una altura de 472 m.s.n.m. con un brillo de 720 luz. Está situado a $4 \mathrm{~km}$ del casco urbano del municipio de San José del fragua, se encuentra cerca al piedemonte de la Cordillera Oriental y al nororiente del Municipio de San José del Fragua. En la zona de amortiguación al Parque Nacional Natural Alto Fragua Indi Wasi. Cuenta con un clima cálido húmedo $\left(\mathrm{T}>25^{\circ} \mathrm{C}\right)$, precipitación $>$ a $2751 \mathrm{~mm}$, con una tendencia monomodal con los mayores picos de precipitación en mayo y junio, y meses secos en enero y febrero, Humedad relativa del $90 \%$, brillo solar 4 horas diarias de luz solar. El proyecto se desarrolló en los bosques alrededor de la Institución Educativa y en la quebrada la oruga (Figura 1).

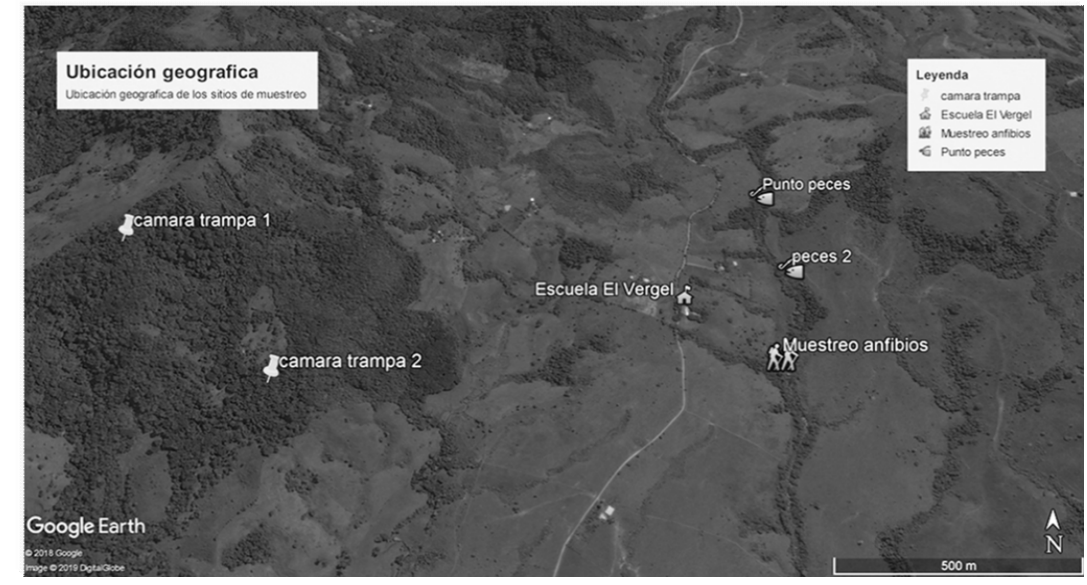

Figura 1. Ubicación de las cámaras trampas y los sitios de muestreo en la Sede el Vergel de la Institución Educativa Rural Platanillo en el municipio de San José del Fragua.

Tipo de Investigación: Para el desarrollo de este proyecto, se usó una investigación cuantitativa que permitió colectar información de los inventarios de biodiversidad en el área de ciencias naturales de los estudiantes. Este tipo de investigación permite la recopilación de datos numéricos de las pruebas diagnósticas que se realicen al grupo de estudiantes para establecer el nivel de competencias en el área de ciencias naturales. Esta investigación es descriptiva teniendo en cuenta la definición propuesta por Castaño (1998): La investigación de tipo descriptiva trabaja sobre realidades de hechos, y su característica fundamental es la de presentar una interpretación correcta (Cardozo, 1997). Para la investigación descriptiva, su preocupación primordial radica en descubrir algunas características fundamentales de conjuntos homogéneos de fenómenos, utilizando criterios 
sistemáticos que permitan poner de manifiesto su estructura o comportamiento. De esta forma se pueden obtener las notas que caracterizan el bosque estudiado (Carvajal Cogollo, 2008).

Puntos de observación en aves: Fueron registradas de manera visual, auditiva. Se utilizó el método de transecto lineal de $1 \mathrm{~km}$, donde se ubicaron puntos de observación distanciados (Ramirez et al. 2004, Renjifo, 2002, Rodriguez, 2002). En cada punto se registraran todos los individuos por especie de ave, que se observe o se escuche, en un radio de $50 \mathrm{~m}$ alrededor del punto durante 15 minutos. Los transecto fueron muestreados en las horas de la mañana iniciando a las 06:00 horas hasta las 10:00 horas y en la tarde desde las 15:00 pm hasta las 18:00 pm. Ésta combinación permitirá una máxima eficiencia y efectividad en los muestreos (Hilty, 2010; Salaman, 2009). Para la taxonomía seguimos la propuesta hecha por Hilty (2001) y Gallo (2005) a nivel de orden, familia, género, para los nombres en lengua tuvimos en cuenta los nombres de los abuelos y de las personas conocedoras de la zona. Para las observaciones utilizamos binoculares. Estas fueron apoyadas en la medida de lo posible mediante la toma de registros fotográficos con cámaras digitales siguiendo a Sanchez(2015).

Mamíferos: Para conocer los mamíferos se realizaron caminatas diurnas y recorridos nocturnos cerca a los caminos en el bosque. En cada encuentro directo con un individuo o grupo de mamíferos registraremos la especie, tamaño de grupo y las coordenadas respectivas. Además, utilizaremos métodos indirectos como huellas, heces, madrigueras, rasguños en los árboles, vocalizaciones y otros rastros que indiquen la presencia de mamíferos (Aquino et al. 2001, Barnett, 1995). Para la identificación de los registros directos e indirectos usaremos las guías de Emmons (1999), Defler (2003), Defler (2010) y Castaño Mora (2002). La taxonomía utilizada será la que sigue a Fototrampeo. Se instalaron 2 cámaras trampas en el bosque. Las trampas cámara cuentan con un sensor de calor y movimiento, por lo que se activan cuando un animal cruza por delante de la cámara (Silver, 2004). Las trampas fueron programadas para tomar fotos de manera continua (24h al día) activadas por la presencia de animales, con un intervalo de 15 segundos entre cada fotografía para de esta manera maximizar el número de fotografías por detección. Las trampas serán colocadas a una altura de 30-50 cm sobre el nivel del suelo dependiendo del sitio de muestreo, activas por 1 a 8 periodos de 24 horas (Simmons, 2010; Kelly 2008).

Peces: Como método de captura se emplearán anzuelos, mayas y se documentarán aquellos métodos artesanales utilizados por la comunidad. En cada sector se tomarán muestras utilizando simultáneamente atarrayas, tres líneas de anzuelos y atarraya durante un período de dos horas de pesca. Las muestras se colectaran durante tres horarios de pesca: de 8:00 a 10:00 (mañana), de 15:00 a 17:00 (tarde) (Jacome, 2003).

Análisis de la información: Se analizaron de forma descriptiva los datos obtenidos con los diferentes instrumentos, a través de tablas estadísticas y gráficas de barras que los niños pudieron interpretar en elárea de tecnología y ciencias naturales

\section{RESULTADOS Y DISCUSIÓN}

La fauna de la zona está poco conocida en términos generales, hasta el momento no se cuenta con un inventario actualizado ni se han hecho evaluaciones ecológicas, lo que se tiene son los registros alrededor del Parque Natural Alto Fragua India Wasi esta investigación es el primer acercamiento a 
un inventario de la zona, que mediante durante los recorridos con los niños fue posible realizar observaciones directas e indirectas de fauna silvestre como son (plumas, pieles, huesos, huellas , madrigueras y fototrampeo). Se encontró o un total de 81 especies registradas donde el grupo taxonómico con el mayor número de especies fue el de aves con 56 especies, seguido de los mamíferos y en último lugar anfibios, reptiles y peces. Esto se debe al mayor número de especies y los hábitos de esta clase, que facilitan su visualización. El grupo taxonómico que concentro la mayor riqueza de especies fue la clase correspondiente a la de aves con un $67 \%$ de las especies, seguido de la clase de los mamíferos, anfibios, peces y reptiles. Durante el inventario registramos 54 aves, 6 anfibios, 5 reptiles, 11 mamíferos y 5 peces (Figura 2). Se estiman que para la zona se estiman 1287 especies de plantas vasculares y hasta 700 especies de vertebrados para la región.

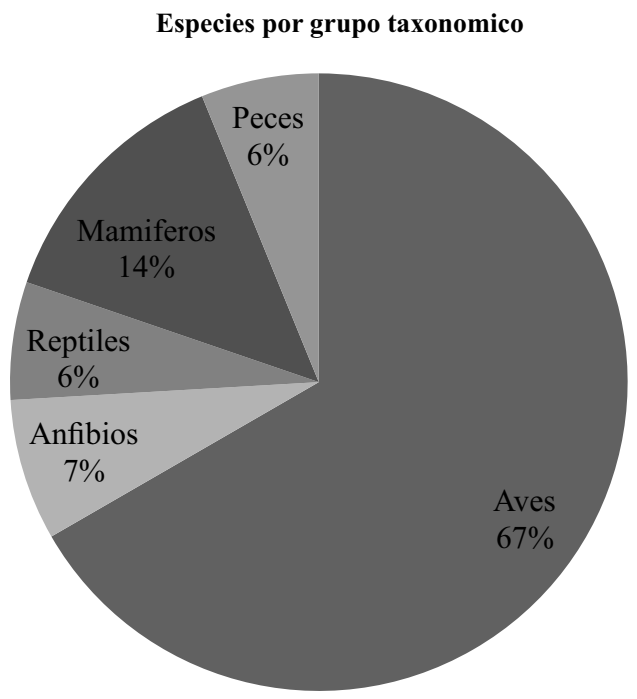

Figura 2. División porcentual de grupos taxonómicos encontrados en la investigación.

Aves: Las aves son el grupo biológico de vertebrados, más conocidos en estos últimos tiempos mediante amplios estudios, debido a que son muy representativas en el planeta presentando la riqueza más alta en los estudios de biodiversidad y muestran varios tipos de respuestas a las perturbaciones de su entorno (Stiles, 2000 y Lima, 1999). Ellas cumplen un papel importante como bioindicador del estado de conservación de una zona determinada como un bosque ripario, de galería o un rastrojo. De las especies más representativas que se pudo observar en la zona y áreas adyacentes por tener fuentes de alimentación en la zona fueron todo el grupo de aves cantoras como son las paseriformes, guacharacas (cracidos), psitácidos (loros), gallinazos (Coragyps atratus), guacamaya cariseca (Ara severus), lora Cabeciamarilla (Amazona ochrocephala) entre otros. Fueron registradas un total de 54 especies de aves.

El orden más representativo fue el orden de los paseriformes con 10 especies (Figura 3), coincidiendo con los reportes de Valencia, et al. (2005) para el departamento del Caquetá, debido a que los bosques satisfacen su alta demanda de alimentos que consisten en frutos, suplementada en cantidades variables de insectos; y estas a su vez son indicadoras de calidad de hábitat dado la gran dependencia que presentan estas poblaciones a la composición y estructura de la vegetación (Stiles y Rosselli 2010; McMulla et al. 2011). 


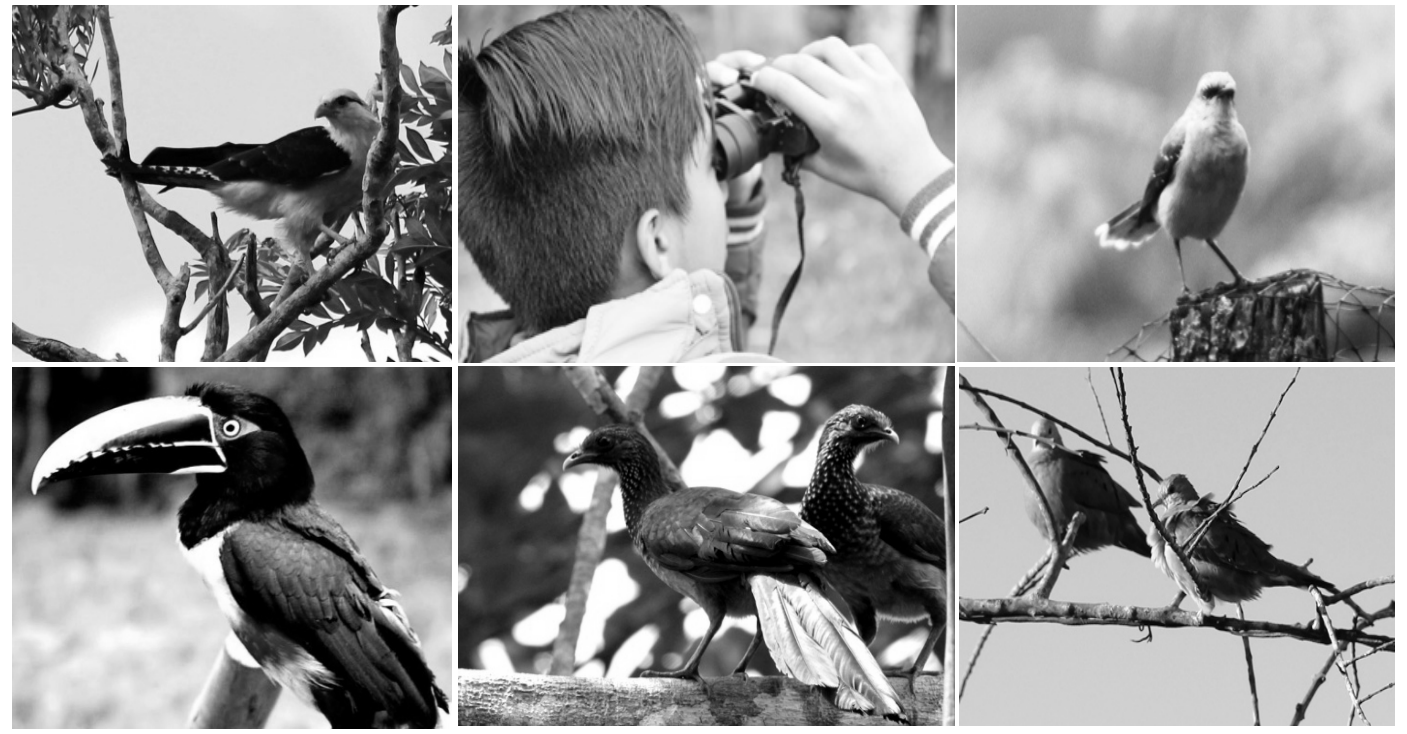

Figura 3. Aves observadas mediante la metodología de puntos de observación.

Mamíferos: Se encontró un total de 11 mamíferos; el bajo número de especies hallado pudo estar asociado a dos razones, primero los procesos de deforestación ocasionados por la presión antrópica ha provocado una reacción en cadena de reducción considerable de algunas de las poblaciones de mamíferos ya que, si no hay bosque, no tienen sostén de supervivencia, en pocas palabras se está rompiendo el ecosistema, la segunda razón el grupo de investigación estuvo conformado por 25 estudiantes donde el ruido inevitable influye sobre el esfuerzo de muestreo. A pesar de las razones anteriormente expuestas y gracias a los métodos no invasivos utilizados se pudo registrar mediante trampas cámara y observaciones de primates como lo fueron: Saimiri macrodon (mono ardilla), guaras, armadillos y marsupiales como las chuchas coincidiendo con los reportes de Voss (2001) para bosques fragmentados (Figura 4).
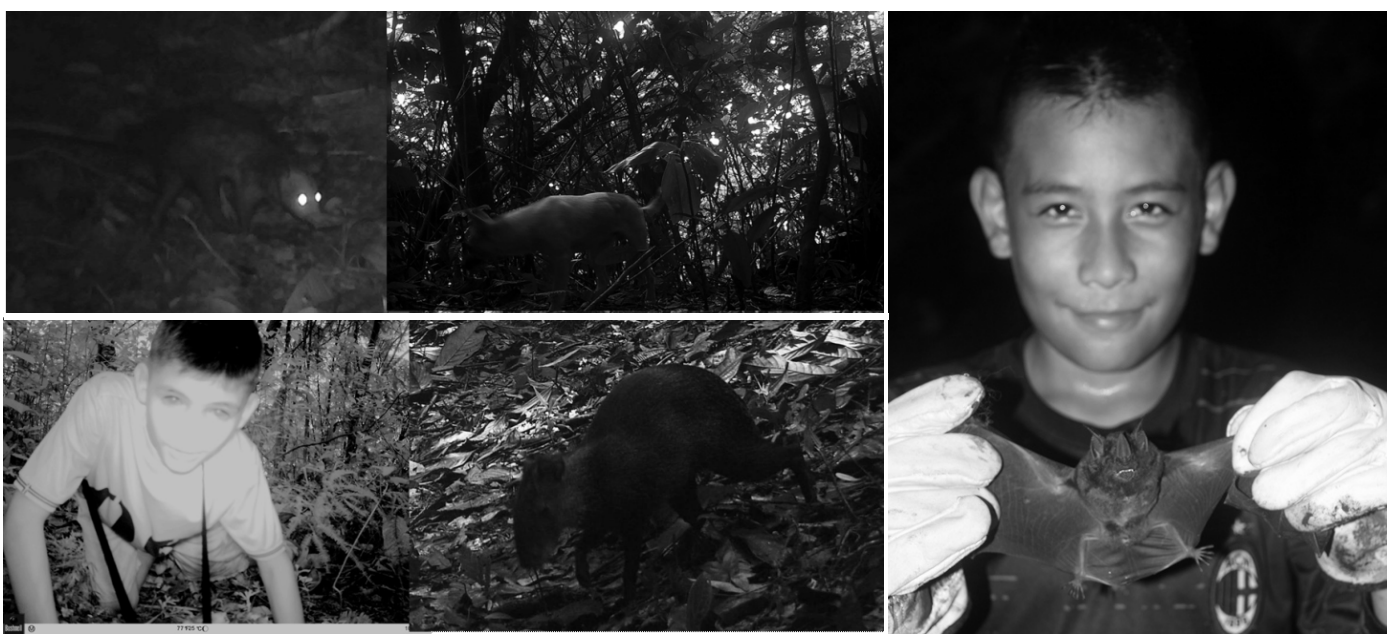

Figura 4. Mamíferos no voladores y voladores mediante técnicas como fototrampeo y búsqueda intensiva de huellas. 
Reptiles: Los resultados encontrados fueron los primeros registros de anfibios y reptiles para la zona. El Papel que juegan los anfibios y reptiles en los bosques son los de regular las redes tróficas en los bosques, en el caso de los anfibios son los únicos vertebrados que tienen la capacidad de transportar energía del medio acuático al terrestre, ya que ocupamos el tercer puesto en biodiversidad de vertebrados y el tercero en anfibios. En la actualidad las poblaciones de anfibios y reptiles se ven afectadas por los diversos factores que afectan los ecosistemas, las presiones antrópicas tal como el calentamiento global y la perdida de cobertura vegetal ocasionando pérdidas de cuerpos de agua en los cuales proliferan dichos organismos, fragmentación de habitad y enfermedades propias de los organismos. Entre las especies de anfibios podemos encontrar los Sapos comunes (Bufo spp), las ranas de laguna (Pristimantis), ranas venenosas del género Ameerega y ranas cafés como lo son Hypsiboas cinerascens. Para reptiles se pueden encontrar serpientes venenosas como la o pelo de gato (Bothrops atrox), Talla X, corales (Micrurus) y otras no venenosas como el güio (Boa constrictor). Entre otros lagartos como son: Anolis planiceps y algunos gecónidos (Figura 5).

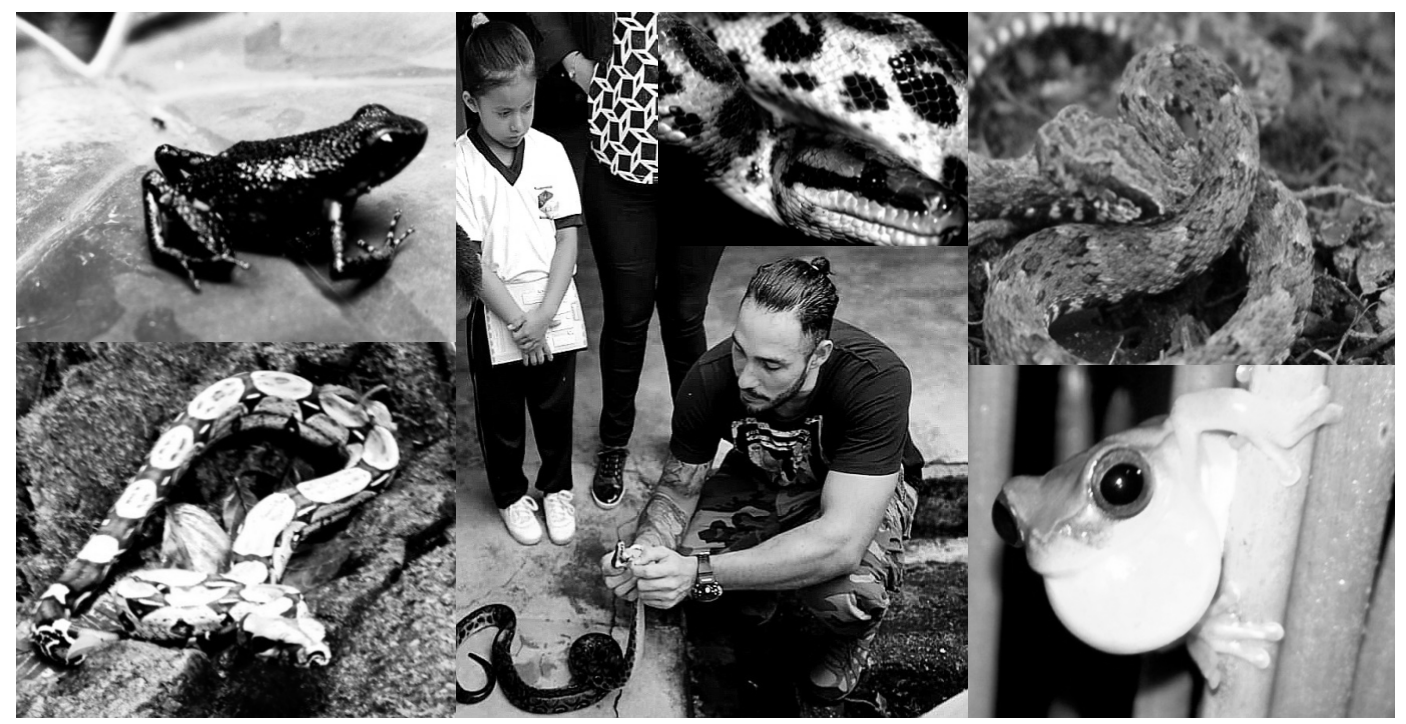

Figura 5. Especies de anfibios y reptiles registradas durante la investigación. De izquierda a derecha rana venenosa (Ameerega sp.), boa esmeralda (Epicrates cenchria), pelo de gato (Bothrops atrox), boa constrictor y rana de la familia Hylidae.

Peces: EL agua de la quebrada la oruga se caracterizó por ser de tipo clara de coloración transparente, con $\mathrm{pH}$, ligeramente ácido y pobreza en nutrientes disueltos, la biota acuática tiene similaridad con la que habita en aguas negras. El muestreo arrojo especies de peces adaptadas a estas condiciones como son los Loricaridos (Cuchas) y las sardinas.

\section{CONCLUSIONES}

Los datos presentados en esta investigación corresponden al primer listado actualizado de biodiversidad faunística para el sur de la amazonia Caqueteña donde se encontró o un total de 81 especies registradas donde el grupo taxonómico con el mayor número de especies fue el de aves con 56 especies, seguido de los mamíferos y en último lugar anfibios, reptiles y peces. Esto se debe al 
mayor número de especies y los hábitos de esta clase, que facilitan su visualización.

Con la implementación del proyecto de investigación se remplazó la metodología tradicional dentro del aula de clase, utilizando herramientas tecnológicas (binoculares, trampas cámara, binoculares y termohigrómetros) como estrategias didácticas e innovadoras en el desarrollo de las actividades académicas con el fin de fortalecer los procesos de enseñanza aprendizaje de las ciencias naturales. Lo anterior, permitió a los estudiantes integrar los componentes del área de ciencias naturales mediante experiencias significativas con los inventarios de biodiversidad, fortalecer las competencias científicas, compartir información de manera didáctica y promover la exploración por el ecosistema de la región rural de san José de la fragua.

\section{LITERATURA CITADA}

AQUINO, R., et al. Mamíferos de la cuenca del Río Samiria, Ecología Poblacional y Sustentabilidad de la Caza. Junglevagt for Amazonas AIF-WWF/DK y Wildlife Conservation Society. Lima-Perú. 2001.Pp.116

BARNETT A, DUTTON J. Expedition Field Techniques: Small Mammals (excluding bats) 2nd edition. Royal Geographic Soviet watt IBG, London. 1995. Pp. 131

CARDOZO, A. El convite: una metodología alternativa para procesos participativos de desarrollo rural sostenible. En: Convirtiendo Portuguesa. Caracas: Editorial CENDI-PROGIS. 1997.Pp.134

CARVAJAL COGOLLO, J. Patrones de diversidad y composición de reptiles en fragmentos de bosque seco tropical en Córdoba, Colombia. Tropical Conservación Science. 2008. Vol. 1(4): Pp. 397-416.

CASTAÑO, J G. Inventario preliminar de aves y mamíferos presentes en fragmentos boscosos en el medio San Jorge, Departamento de Córdoba. Crónica Forestal y medio ambiente. Vol. 13. Número 1. Universidad Nacional de Colombia. 1998. Pp. 45

CASTAÑO MORA, O., et al. Libro rojo de reptiles de Colombia. Libros rojos de especies amenazadas de Colombia. Bogotá: Instituto de Ciencias Naturales, Universidad Nacional de Colombia, Ministerio del Medio Ambiente, Conservación Internacional, Colombia.2002. Pp. 268

DEFLER, Thomas. Primates de Colombia. Conservación Internacional. Serie de guías tropicales de campo. Bogotá-Colombia. 2003. Pp. 456

DEFLER, Thomas. Historia natural de los primates Colombianos. Conservación Internacional. 2010.Pp. 456

DERUYTTERE., et al. Pueblos indígenas y desarrollo sostenible: el papel del Banco Interamericano de Desarrollo. Washington D.C. En: Unidad de Pueblos Indígenas y Desarrollo Comunitario, Departamento de Desarrollo Sostenible.1997. Pp.45 
EMMONS, H.L.Mamíferos de los bosques húmedos de América Tropical. Editorial F.A.N. Santa Cruz de la Sierra, Bolivia fragmentación. Conservation .1999. Pp.1124- 1139

GALLO CAJIAO, IM. Fragmentos de bosque y conservación de aves: un estudio de caso en los Andes de Colombia. MEMORIAS: Manejo de Fauna silvestre en Amazonia y Latinoamérica. 2005. Pp.178

GEILFUS, Frans. Ochenta herramientas para el desarrollo participativo. San Salvador: IICA. 1996. Pp.235

GRAIPEL,Mauricio. A simple ground-based method for trapping small mammals in the forest canopy. Mastozoología Neotropical . 2003. Pp. 34

HILTY ,S. Guía de las Aves de Colombia. Traducción al español por Humberto Álvarez López. American Bird Conservancy.2010. Pp.1030.

JÁCOME NEGRETE, I. Etnoictiología Kichwa de las lagunas de la cuenca baja del río Curaray (Amazonia), Ecuador Biota Colombiana.2003.Pp. 45

KELLY, MJ. Camera Trapping of Carnivores: Trap Success Among Camera Types and Across Species, and Habitat Selection by Species, on Salt Pond Mountain, Giles County, Virginia. 2008.Pp. 249-262

LIMA, MG.The conservation value of linear forest remnants in central Amazonia. Biological Conservation 91. 1999. Pp. 241-247

MEN.Ministerio de Educación Nacional. Saber 3, 5 y 9: resultados nacionales 2009-2014. Bogotá: El Ministerio. 2015.34 p.

MCMULLAN, M., et al. Guía de campo de las aves de Colombia. Proaves. Colombia. 2011. Pp. 245

RAMÍREZ, B., et al. Aporte al conocimiento y sostenibilidad del agroecosistema intervenido de la Amazonia colombiana. Universidad de la Amazonia.Colombia.2004. Pp. 45

RENJIFO, L., et al. Libro Rojo De Aves De Investigación En Recursos Biológicos Alexander Von Humboldt, Bogotá, Colombia. 2002.Pp.506

RODRÍGUEZ MAHECHA, J., et al. Loros, pericos y guacamayas neotropicales. Bogotá: Conservación Internacional Serie Libreta de Campo.2005. Pp.189

SALAMAN, P., \& DONEGAN, T. Listado de las Aves de Colombia 2008. Conservación Colombiana. 2009. Pp. 89

SANCHEZ, L.R. Monitoreo comunitario de aves: un elemento fundamental en los esfuerzos de conservación de la biodiversidad", in Plumas de multitudes, integración comunitaria en el estudio y 
monitoreo de aves en México, Ortega-Álvarez, R., L. SÁNCHEZ GONZÁLEZ \& H. BERLANGAGARCÍA. Eds. México: Comisión Nacional para el Conocimiento y Uso de la Biodiversidad, 2015. Pp. 13-22.

SILVER, S. Estimando la Abundancia de Jaguares Mediante Trampas-Cámara. W. C. Society. 2004. Pp. 34

SIMMONS, N B. The Mammals of Paracou, French Guiana: A Neotropical Lowland Rainforest Fauna, Part 1: bats. Bulletin of the American Museum of Natural History. 2010. Pp. 219

STILES BOHORQUEZ, C I. Evaluando el estado de la Biodiversidad: el caso de la avifauna de la serranía de las Quinchas, Boyacá, Colombia. Caldasia. 2000. Pp. 61-92

STILES F. G. \& ROSSELLI L. Inventario de aves de un bosque andino: Comparación de dos métodos. Caldasia. 2010. 1998. Pp. 29-43

VALENCIA, A., et al. Lista anotada de las aves de los humedales de la parte alta del departamento de Caquetá. 2005.Pp. 34

VOSS, L. The Mammals of Paracou, French Guiana : A Neotropical lowland Rainforest Fauna. Part 2. Nonvolant species Bulletin of The American Museum of Natural History. 2001. Pp. 236-266 